\title{
LONG-TERM MONITORING OF THE HIGH-ENERGY $\gamma$-RAY EMISSION FROM LS I +61³03 AND LS 5039
}

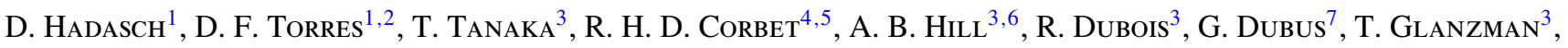

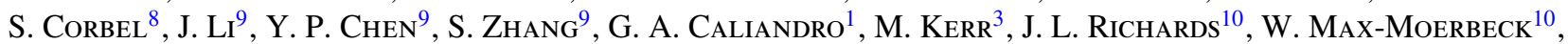 \\ A. READHEAD ${ }^{10}$, AND G. POOLEY ${ }^{11}$ \\ ${ }^{1}$ Institut de Ciències de l'Espai (IEEC-CSIC), Campus UAB, Torre C5, 2a planta, 08193 Barcelona, Spain \\ ${ }^{2}$ Institució Catalana de Recerca i Estudis Avançats (ICREA), 08010 Barcelona, Spain \\ ${ }^{3}$ W. W. Hansen Experimental Physics Laboratory, Kavli Institute for Particle Astrophysics and Cosmology, Department of Physics \\ and SLAC National Accelerator Laboratory, Stanford University, Stanford, CA 94305, USA \\ ${ }^{4}$ NASA Goddard Space Flight Center, Greenbelt, MD 20771, USA \\ ${ }^{5}$ Center for Space Science and Technology, University of Maryland Baltimore County, Baltimore, MD 21250, USA \\ ${ }^{6}$ School of Physics and Astronomy, University of Southampton, Highfield, Southampton SO17 1BJ, UK \\ ${ }^{7}$ UJF-Grenoble 1/CNRS-INSU, Institut de Planétologie et d'Astrophysique de Grenoble, UMR 5274, 38041 Grenoble, France \\ ${ }^{8}$ Université Paris 7 Denis Diderot and Service d'Astrophysique, UMR AIM, CEA Saclay, F-91191 Gif sur Yvette, France \\ ${ }^{9}$ Key Laboratory of Particle Astrophysics, Institute of High Energy Physics, Chinese Academy of Science, Beijing 100049, China \\ ${ }^{10}$ Cahill Center for Astronomy and Astrophysics, California Institute of Technology, Pasadena, CA 91125, USA \\ ${ }^{11}$ Cavendish Laboratory, Cambridge CB3 OHE, UK \\ Received 2011 November 11; accepted 2012 February 6; published 2012 March 22
}

\begin{abstract}
The Fermi Large Area Telescope (LAT) reported the first definitive GeV detections of the binaries $\mathrm{LS} \mathrm{I}+61^{\circ} 303$ and LS 5039 in the first year after its launch in 2008 June. These detections were unambiguous as a consequence of the reduced positional uncertainty and the detection of modulated $\gamma$-ray emission on the corresponding orbital periods. An analysis of new data from the LAT, comprising 30 months of observations, identifies a change in the $\gamma$-ray behavior of LS I $+61^{\circ} 303$. An increase in flux is detected in 2009 March and a steady decline in the orbital flux modulation is observed. Significant emission up to $30 \mathrm{GeV}$ is detected by the LAT; prior data sets led to upper limits only. Contemporaneous $\mathrm{TeV}$ observations no longer detected the source, or found it—in one orbit—close to periastron, far from the phases at which the source previously appeared at $\mathrm{TeV}$ energies. The detailed numerical simulations and models that exist within the literature do not predict or explain many of these features now observed at $\mathrm{GeV}$ and $\mathrm{TeV}$ energies. New ideas and models are needed to fully explain and understand this behavior. A detailed phase-resolved analysis of the spectral characterization of LS I $+61^{\circ} 303$ in the GeV regime ascribes a power law with an exponential cutoff spectrum along each analyzed portion of the system's orbit. The on-source exposure of LS 5039 is also substantially increased with respect to our prior publication. In this case, whereas the general $\gamma$-ray properties remain consistent, the increased statistics of the current data set allows for a deeper investigation of its orbital and spectral evolution.
\end{abstract}

Key words: binaries: close - gamma rays: general - stars: variables: general - X-rays: binaries - X-rays: individual (LS I +61 303) - X-rays: individual (LS 5039)

Online-only material: color figures

\section{INTRODUCTION}

To date there are only a handful of X-ray binaries that have been detected at high (HE; $0.1-100 \mathrm{GeV})$ or very high energies $\left(>100 \mathrm{GeV}\right.$ ): $\mathrm{LS} \mathrm{I}+61^{\circ} 303$ (Albert et al. 2006; Acciari et al. 2008; Abdo et al. 2009a), LS 5039 (Aharonian et al. 2005b; Abdo et al. 2009b), PSR B1259-63 (Aharonian et al. 2005a; Abdo et al. 2011; Tam et al. 2011), Cyg X-3 (Abdo et al. 2009c), and Cyg X-1 (Albert et al. 2007; Sabatini et al. 2010). Recently, two new binaries were found: 1FGL J1018.6-5856, with a period of 16.6 days found in the $\mathrm{GeV}$ regime (Corbet et al. 2012) and HESS J0632+057 (Falcone et al. 2011; Ong 2011; Mariotti 2011), for which a period of $\sim 320$ days was detected in X-rays (Bongiorno et al. 2011). Of these sources only LS I +61 ${ }^{\circ} 303$, LS 5039, and PSR B1259-63 share the property of being binaries detected at both $\mathrm{GeV}$ and $\mathrm{TeV}$ energies. The other systems have been unambiguously detected only in one band, either at $\mathrm{GeV}$ or at $\mathrm{TeV}$, see, e.g., the case of Cyg X-3 in Aleksić et al. (2010). In the case of Cyg X-1, with the hint of $\mathrm{TeV}$ detection itself being at the level of four standard deviations $(4 \sigma)$, claims of detection at $\mathrm{GeV}$ energies by the Astrorivelatore Gamma a Immagini Leggero remain uncertain with concurrent
Fermi Large Area Telescope (LAT) observations (Hill et al. 2011). It is yet uncertain whether these spectral energy distribution (SED) differences reflect an underlying distinct nature, or are just a variability signature in different bands.

The nature of the binary compact object in LS I $+61^{\circ} 303$, LS 5039, HESS J0632+057, and 1FGL J1018.6-5856 is as yet undetermined (Hill et al. 2011). Both neutron star (e.g., PSR B1259-63) and probable black hole (e.g., Cyg X-3) binary systems have been detected at $\mathrm{GeV}$ energies, and so both types of compact object are viable in the undetermined systems. Recently, the Burst Alert Telescope on board Swift reported a magnetar-like event which may have emanated from LS I +61 303 (Barthelmy et al. 2008; Torres et al. 2012). If true, then this would be the first magnetar found in a binary system.

The early LAT reports of GeV emission from LS 5039 and LS I $+61^{\circ} 303$ were based upon 6-9 months of survey observations (Abdo et al. 2009a, 2009b). Both sources were detected at high significance and were unambiguously identified with the binaries by their flux modulation at the corresponding orbital periods, 26.4960 days for LS I $+61^{\circ} 303$ (Gregory 2002) and 3.90603 days for LS 5039 (Casares et al. 2005). The modulation patterns were roughly consistent with expectations from 
inverse Compton scattering plus $\gamma-\gamma$ absorption models, and were anti-correlated in phase with pre-existing $\mathrm{TeV}$ measurements (e.g., Albert et al. 2009; Aharonian et al. 2006). The anti-correlation of $\mathrm{GeV}-\mathrm{TeV}$ fluxes is, in fact, a generic feature of these models, where the $\mathrm{GeV}$ emission is enhanced (reduced) when the highly relativistic electrons seen by the observer encounter the seed photons head-on (rear-on) (e.g., see Bednarek 2007; Sierpowska-Bartosik \& Torres 2007; Dubus et al. 2008; Khangulyan et al. 2008). Fermi-LAT measurements provided a confirmation of these predictions.

The spectra of both sources were best modeled with exponential cutoffs in their HE spectra, at least along part of the orbit. Specifically, an exponential cutoff was statistically a better fit to the SED compared with a pure power law at phases surrounding the superior conjunction (SUPC) of LS 5039 and in the orbitally averaged spectrum of LS I $+61^{\circ} 303$. Statistical limitations of the data prevented the determination or the ruling out of an exponential cutoff in any part of the orbit of LS I $+61^{\circ} 303$ or in the inferior conjunction (INFC) of LS 5039. The SEDs with the exponential cutoffs that were reported were reminiscent of the many pulsars the LAT has detected since launch (Abdo et al. 2009 b), although this was far from a proof of their pulsar nature. To date no pulsations have been found at $\mathrm{GeV}$ energies, or at any other wavelengths, despite deep dedicated searches (see, e.g., Rea et al. 2010, 2011).

Since Fermi was launched, both the Major Atmospheric Gamma-ray Imaging Cherenkov Telescopes (MAGIC) and the Very Energetic Radiation Imaging Telescope Array (VERITAS) have performed observations of $\mathrm{LS} \mathrm{I}+61^{\circ} 303$. No TeV detection was reported after 2008 October, until the source unexpectedly reappeared, once, at periastron (Acciari et al. 2011; Ong 2010). At the same time, a hard X-ray multi-year analysis (Zhang et al. 2010) and a long-term X-ray campaign on LS I +61 303 using the Rossi X-ray Timing Explorer (RXTE) has been conducted covering the whole extent of the LAT observations (see Torres et al. 2010; Li et al. 2011). In addition, simultaneous and archival data from long-term monitoring of radio and $\mathrm{H} \alpha$ emission is available for comparison in a multi-wavelength context. In this work, we present the results of the analysis of 30 months of LAT survey observations of both LS I $+61^{\circ} 303$ and LS 5039. We investigate the long-term flux variations of the sources, as well as variations in the amplitude of their orbital flux modulation, and we explore the possible spectral variability for both systems, finally putting and interpreting these observations in the context of the source behavior at other frequencies.

\section{OBSERVATIONS AND DATA REDUCTION}

The Fermi-LAT is an electron-positron pair production telescope, featuring solid state silicon trackers and cesium iodide calorimeters, sensitive to photons from $\sim 20 \mathrm{MeV}$ to $>300 \mathrm{GeV}$ (Atwood et al. 2009). It has a large $\sim 2.4$ sr field of view (at $1 \mathrm{GeV}$ ) and an effective area of $\sim 8000 \mathrm{~cm}^{2}$ for $>1 \mathrm{GeV}$.

\subsection{Data Set}

The Fermi survey mode operations began on 2008 August 4; in this mode, the observatory is rocked north and south on alternate orbits to provide a more uniform coverage, so that every part of the sky is usually observed for $\sim 30$ minutes every $\sim 3 \mathrm{hr}$. Therefore, the two sources of interest were monitored regularly without significant breaks, allowing us to draw a complete picture of their behavior in $\gamma$-rays over the last
2 years. The data set used for this analysis spans 2008 August 4 through 2011 January 24.

The data were reduced and analyzed using the FERMI SCIENCE ToOLs v9R20 package. $^{12}$ The standard on board filtering, event reconstruction, and classification were applied to the data (Atwood et al. 2009). The high-quality "diffuse" event class was used together with the "Pass 6 v3 Diffuse" instrument response functions (IRFs). Time periods when the target source was observed at a zenith angle greater than $105^{\circ}$ were excluded to limit contamination from Earth limb photons. Where required in the analysis, models for the Galactic diffuse emission ( $\left.g l l \_i e m \_v 02 . f i t\right)$ and isotropic backgrounds (isotropic_iem_v02.txt) were used. ${ }^{13}$

\subsection{Spectral Analysis Methods}

The binned maximum likelihood method of gtlike, included in the Science Tools, was used to determine the intensities and spectral parameters presented in this paper. We used all photons with energy $>100 \mathrm{MeV}$ in a circular region of interest (ROI) of $10^{\circ}$ radius centered at the position of LS I $+61^{\circ} 303$ and LS 5039, respectively. For source modeling, the 1FGL catalog (Abdo et al. 2010a), derived from 11 months of survey data, and the first Fermi pulsar catalog (Abdo et al. 2010b) were used; all sources within $15^{\circ}$ of the ROI center were included. The energy spectra of point sources included in the catalog within our ROI are modeled by a simple power law,

$$
\frac{d N}{d E}=N_{0}\left(\frac{E}{E_{0}}\right)^{-\Gamma}
$$

with the exception of known $\gamma$-ray pulsars, which were modeled by power laws with exponential cutoffs described by

$$
\frac{d N}{d E}=N_{0}\left(\frac{E}{E_{0}}\right)^{-\Gamma} \exp \left[-\left(\frac{E}{E_{\text {cutoff }}}\right)\right] .
$$

The spectral parameters were fixed to the catalog values, except for the sources within $3 \mathrm{deg}$ of the candidate location. For these latter sources, the flux normalization was left free. All of the spectral parameters of the two subject binaries were left free for the fit. Source detection significance is determined using the Test Statistic value, $T S=-2 \ln \left(L_{0} / L_{1}\right)$ which compares the likelihood ratio of models including, e.g., an additional source, with the null hypothesis of background only (Mattox et al. 1996).

To estimate the systematic errors, which are mainly caused by uncertainties in the effective area and energy response of the LAT as well as background contamination, we use the socalled bracketing IRFs. These are IRFs with effective areas that bracket those of our nominal IRF above and below by linearly connecting differences of $(10 \%, 5 \%, 20 \%)$ at $\log (E / \mathrm{MeV})$ of $(2,2.75,4)$, respectively.

\subsection{Timing Analysis Methods}

Light curves are extracted using aperture photometry, taking an aperture radius of $1^{\circ}$ and using the gtbin tool. The exposure correction is performed with the tool gtexposure assuming the spectral shape of the source to be a power law with

\footnotetext{
${ }^{12}$ See the Fermi Space Science Center (FSSC) Web site for details of the Science Tools: http://fermi.gsfc.nasa.gov/ssc/data/analysis/.

${ }^{13}$ Descriptions of the models are available from the FSSC: http://fermi.gsfc.nasa.gov/ssc.
} 

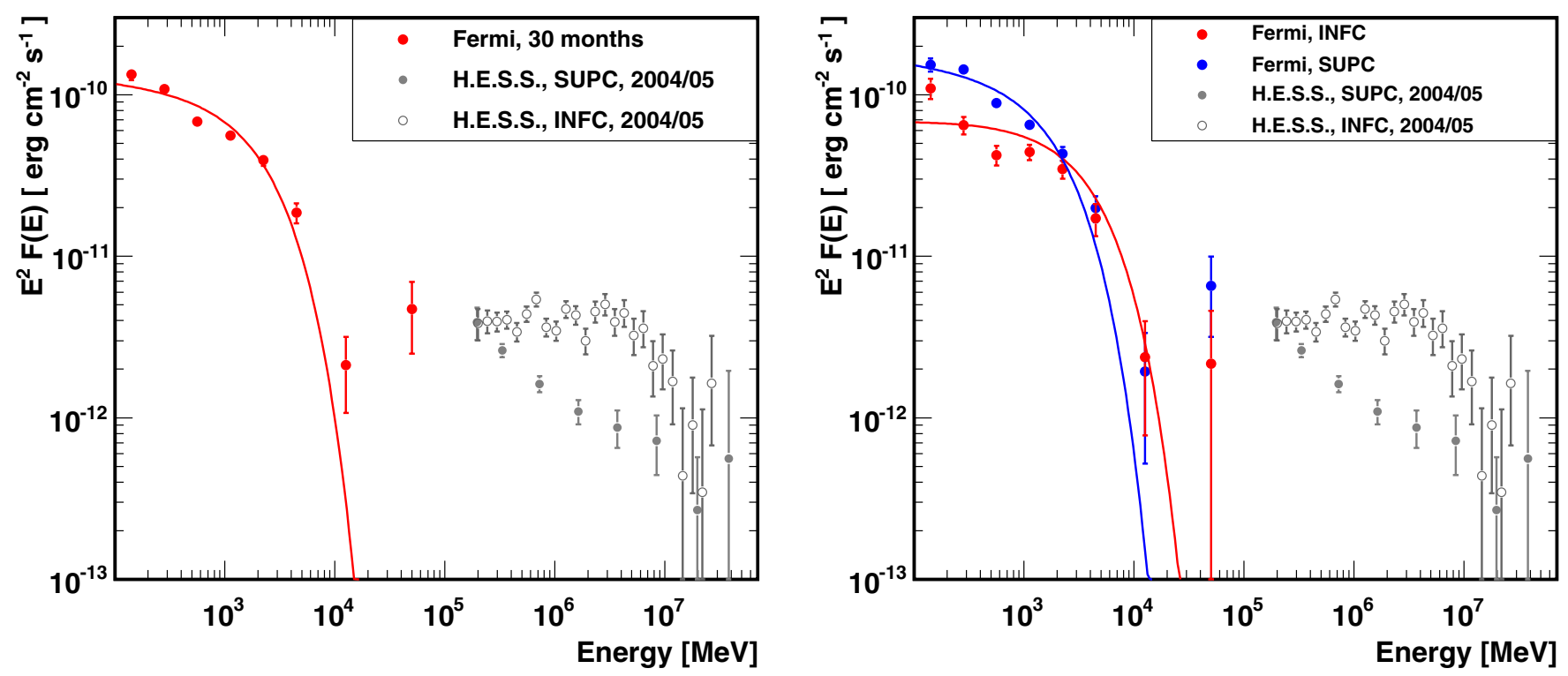

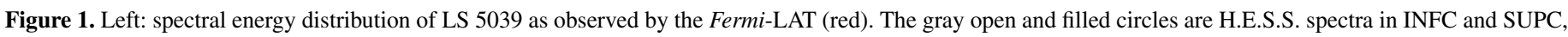

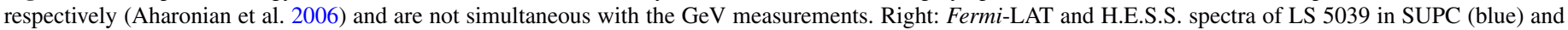
INFC (red).

(A color version of this figure is available in the online journal.)

an exponential cutoff (see Sections 3.1 and 4.1). These light curves are not background subtracted. The folded light curves shown in the subsequent sections are derived by performing gtlike fits for each phase bin. Therefore, all of them are effectively background subtracted. We check that both methods for generating light curves, aperture photometry, and gtlike fits are consistent with each other when the former light curves are background subtracted too.

The primary method of timing analysis employed searches for periodic modulation by calculating the weighted periodogram of the light curve (Lomb 1976; Scargle 1982; Corbet \& Dubois 2007). The light curve is constructed by summing, for each photon, the estimated probability that the photon came from the source of interest. The probability will be both spatially and spectrally dependent. Because this technique allows for the correct weighting of each photon, it intrinsically improves the signal-to-noise and allows the use of a larger aperture. This method has successfully been applied to increase the LAT sensitivity for the detection of pulsars (Kerr 2011). However, in the basic form of this technique, the weight for any particular energy/position is fixed. This means that changes in source brightness will not be reflected in the weights and can result in incorrect probabilities. The calculation of probabilities was performed using the tool gtsrcprob and the same source model file derived from the 1FGL catalog and used in the spectral analysis. Since the exposure of the time bins was variable, the contribution of each time bin to the power spectrum was weighted based on its relative exposure. Period errors are calculated using the method of Horne \& Baliunas (1986).

\section{LS 5039 RESULTS}

LS 5039 is located in a complicated region toward the inner Galaxy with high Galactic diffuse emission and many surrounding $\gamma$-ray sources. In particular, the LAT detected a bright $\left(8.7 \times 10^{-7}\right.$ photons $\mathrm{cm}^{-2} \mathrm{~s}^{-1}$ above $\left.100 \mathrm{MeV}\right) \gamma$-ray pulsar, PSR J1826-1256, $2^{\circ}$ away from LS 5039. Following the analysis performed in the earlier LAT paper (Abdo et al. 2009b), we discarded events whose arrival times correspond to the peaks of the pulsar cycle of PSR J1826-1256 in order to minimize the contamination from the pulsar. The excluded pulse phase of PSR J1826-1256 is $0.05<\phi_{p}<0.2$ and $0.625<\phi_{p}<0.775$ (see Figure 37 in Ray et al. 2011), which results in a loss of $30 \%$ exposure on LS 5039. To account for the loss, a scaling factor of $1 / 0.7$ is multiplied to fluxes obtained with maximum likelihood fits.

\subsection{Orbitally Averaged Spectrum}

The orbitally averaged spectrum of LS 5039 was initially investigated by fitting a power law and a power law with an exponential cutoff to the data. We compare two models utilizing the likelihood ratio test (Mattox et al. 1996), i.e., for the ratio $2 \times \Delta \log$ (Likelihood), we assume a $\chi^{2}$-distribution to calculate the probabilities taking into account the corresponding degrees of freedom (Eadie et al. 1971). In this case, the significance of a spectral cutoff was assessed by comparing the likelihood ratio between the power law and cutoff power-law cases, which is $-2 \ln \left(L_{\mathrm{PL}} / L_{\text {cutoff }}\right)=94.9$, where $L_{\mathrm{PL}}$ and $L_{\text {cutoff }}$ are the likelihood values obtained for the spectral fits with a power law and a cutoff power law, respectively. This indicates that the simple power-law model is rejected at the $9.7 \sigma$ level in favor of the cutoff power law. The best-fit parameters for the cutoff power-law model are $\Gamma=2.06 \pm 0.06_{\text {stat }} \pm 0.11_{\text {syst }}$ and $E_{\text {cutoff }}=2.2 \pm 0.3_{\text {stat }} \pm 0.5_{\text {syst }} \mathrm{GeV}$ with a flux of $F_{>100 \mathrm{MeV}}=\left(6.1 \pm 0.3_{\text {stat }} \pm 2.1_{\text {syst }}\right) \times 10^{-7} \mathrm{~cm}^{-2} \mathrm{~s}^{-1}$ integrated above $100 \mathrm{MeV}$. Using a cutoff power-law spectral model, the maximum likelihood fit yields a test statistic of $T S=1623$ for the LS 5039 detection; equivalent to $\sim 40 \sigma$. We also tried a broken power-law spectral model for LS 5039 in addition to an exponentially cutoff power law. We found that the broken power law gives lower $T S$ values than the exponentially cutoff power-law case.

Spectral points in each energy band were obtained by dividing the data set into separate energy bins and performing maximum likelihood fits for each of them. The resulting SED is plotted in Figure 1 together with the best-fit cutoff power-law model. 


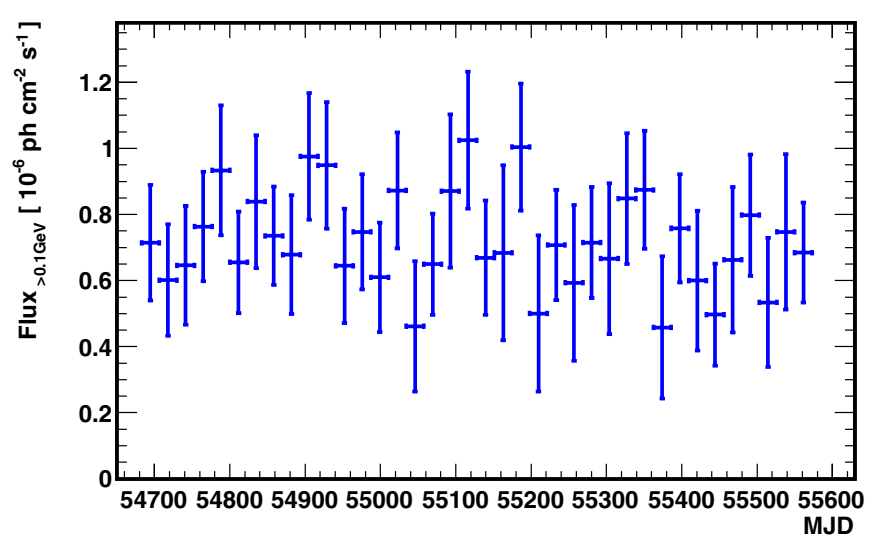

Figure 2. Light curve of LS 5039 in time bins of six orbital cycles between $100 \mathrm{MeV}$ and $300 \mathrm{GeV}$. One orbit is 3.90532 day long.

(A color version of this figure is available in the online journal.)

Interestingly, the SED shows significantly higher flux (one spectral data point) at $\gtrsim 10 \mathrm{GeV}$ than the expected flux from the best-fit cutoff power law, possibly suggesting another component at HEs.

One idea explored for these sources (especially, for LS 5039, see, e.g., Torres 2011) is that the $\gamma$-ray emission could be understood as having two components: one would be the magnetospheric $\mathrm{GeV}$ emission from a putative pulsar and the other from the inter-wind region or from the pulsar wind zone. The latter would be unpulsed and would vary with the orbital phase, the former would be steady and pulsed. The current data for LS 5039 would indeed allow for this possibility, especially because of the possible $\mathrm{HE}$ component found in the $\mathrm{GeV}$ spectrum.

To test the significance of the additional component, we added to the model a power-law source at the location of LS 5039 in addition to the cutoff power-law source. Model A is a power law with a cutoff and model $\mathrm{B}$ a power law with a cutoff plus an additional power law. Thus, model $\mathrm{B}$ has two more free parameters compared to model A. According to the likelihood ratio test, the probability of incorrectly rejecting model $\mathrm{A}$ is $6.1 \times 10^{-6}(4.5 \sigma)$.

The best-fit parameters for the putative additional component are $\Gamma=1.6 \pm 0.4_{\text {stat }} \pm 0.3_{\text {syst }}$ and $F_{>10 \mathrm{GeV}}=\left(1.6 \pm 0.6_{\text {stat }} \pm\right.$ $\left.0.7_{\text {syst }}\right) \times 10^{-10} \mathrm{~cm}^{-2} \mathrm{~s}^{-1}$. The addition of the HE component slightly affects the parameters of the cutoff power law. The bestfit parameters for the latter are $\Gamma=2.02 \pm 0.06_{\text {stat }} \pm 0.10_{\text {syst }}$ and $E_{\text {cutoff }}=2.0 \pm 0.3_{\text {stat }} \pm 0.4_{\text {syst }} \mathrm{GeV}$ with a flux of $F_{>100 \mathrm{MeV}}=\left(6.0 \pm 0.3_{\text {stat }} \pm 1.9_{\text {syst }}\right) \times 10^{-7} \mathrm{~cm}^{-2} \mathrm{~s}^{-1}$ integrated above $100 \mathrm{MeV}$.

\subsection{Phase-resolved Analysis}

Following the H.E.S.S. analysis by Aharonian et al. (2006), as well as the previous one, the whole data set was divided into two orbital intervals: SUPC $(\phi<0.45$ and $0.9<\phi)$ and INFC $(0.45<\phi<0.9)$. The SUPC and INFC data were analyzed in the same way as the orbitally averaged data. Being consistent with our previous paper, the power-law assumption for the SUPC spectrum is rejected with $-2 \ln \left(L_{\mathrm{PL}} / L_{\text {cutoff }}\right)=81.2$, or at a rejection significance of $\sim 9 \sigma$. The best-fit parameters are $\Gamma=$ $2.07 \pm 0.07_{\text {stat }} \pm 0.08_{\text {syst }}, E_{\text {cutoff }}=1.9 \pm 0.3_{\text {stat }} \pm 0.3_{\text {syst }} \mathrm{GeV}$, and $F_{>100 \mathrm{MeV}}=\left(7.8 \pm 0.4_{\text {stat }} \pm 1.9_{\text {syst }}\right) \times 10^{-7} \mathrm{~cm}^{-2} \mathrm{~s}^{-1}$.

Although a single power law was not rejected for INFC in our previous analysis using 10 months of data (Abdo et al.

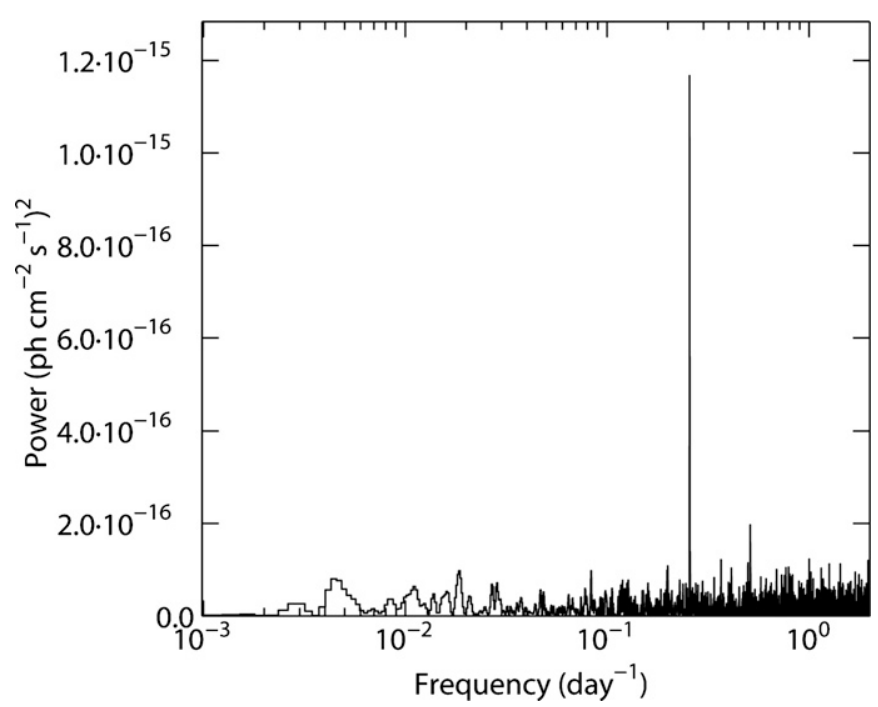

Figure 3. Lomb-Scargle power spectrum of the whole GeV data set on LS 5039.

2009 b), a cutoff power law is preferred also for INFC with the present data set. The likelihood ratio for the INFC data is $-2 \ln \left(L_{\mathrm{PL}} / L_{\text {cutoff }}\right)=21.7$, which corresponds to $4.7 \sigma$. The parameters for the INFC spectrum are $\Gamma=1.99 \pm$ $0.13_{\text {stat }} \pm 0.07_{\text {syst }}, E_{\text {cutoff }}=2.6 \pm 0.7_{\text {stat }} \pm 0.9_{\text {syst }} \mathrm{GeV}$, and $F_{>100 \mathrm{MeV}}=\left(3.9 \pm 0.4_{\text {stat }} \pm 1.5_{\text {syst }}\right) \times 10^{-7} \mathrm{~cm}^{-2} \mathrm{~s}^{-1}$. Therefore, the SUPC and INFC spectral shapes are completely consistent with one another within the errors. The only difference is the normalization and hence the total flux. On the other hand, the spectrum for INFC (red points in the right panel of Figure 1) seems to exhibit additional structure below $1 \mathrm{GeV}$. The limited statistics and the large contribution of diffuse emission at low energies, however, prevents solid conclusions on whether a more complicated fit (e.g., a double broken power law or a broken power law with a cutoff) would be preferred.

We also searched for emission from the HE component in the SUPC and INFC spectra. However, the $T S$ of the additional components compared with a power law with exponential cutoff are only 13.6 and 10.9 for SUPC and INFC, respectively, and do not confirm a second spectral component. The SUPC and INFC SEDs were obtained using the same method as the orbitally averaged spectra and are plotted in the right panel of Figure 1.

\subsection{Light Curve}

Figure 2 shows the light curve for LS 5039 over 30 months derived by performing gtlike fits on time bins which contain six orbital cycles each. The light curve for LS 5039 does not show any significant flux changes. Constructing the periodogram of the weighted photon light curve yields a significant detection of a periodicity at $3.90532 \pm 0.0008$ days. This is consistent with the known orbital period of LS 5039. The Lomb-Scargle power spectrum of LS 5039 is shown in Figure 3. The stability of the orbital modulation was investigated and no significant variation in the modulation fraction as a function of time was found.

\section{LS I +61 ${ }^{\circ} 303$ RESULTS}

\subsection{Orbitally Averaged Spectral Analysis}

We have derived the spectrum of orbitally averaged LAT data, i.e., without any selection criteria (cuts) concerning the orbital phase, for the LS I $+61^{\circ} 303$ system. The spectral points 
Table 1

$T S$ Values for LS I $+61^{\circ} 303$ for Different Spectral Shapes (See Section 4.3 for Details)

\begin{tabular}{|c|c|c|c|}
\hline Data Set & $\begin{array}{c}\text { Power Law }+ \text { Exponential Cutoff } \\
T S\end{array}$ & $\begin{array}{c}\text { Power Law } \\
T S\end{array}$ & $\begin{array}{c}\text { Broken Power Law } \\
T S\end{array}$ \\
\hline 30 months of data & 23995 & 23475 & 23970 \\
\hline Data before 2009 March & 3404 & 3314 & 3415 \\
\hline Data after 2009 March & 20714 & 20283 & 20699 \\
\hline Inferior conjunction (geometrically) & 12548 & 12326 & 12512 \\
\hline Superior conjunction (geometrically) & 11711 & 11422 & 11700 \\
\hline Inferior conjunction (angle cut) & 6670 & 6562 & 6665 \\
\hline Superior conjunction (angle cut) & 6083 & 5986 & 6063 \\
\hline Periastron & 11656 & 11450 & 11636 \\
\hline Apastron & 12377 & 12059 & 12361 \\
\hline
\end{tabular}

Table 2

Parameters for LS I $+61^{\circ} 303$ from Spectral Fitting with Power Law with Exponential Cutoff (See Section 4.3 for Details)

\begin{tabular}{|c|c|c|c|}
\hline Data Set & Photon Index $\Gamma$ & $\begin{array}{l}\text { Cutoff Energy } \\
\qquad(\mathrm{GeV})\end{array}$ & $\begin{array}{c}\text { Flux }>100 \mathrm{MeV} \\
\left(\times 10^{-6} \text { photons } \mathrm{cm}^{-2} \mathrm{~s}^{-1}\right)\end{array}$ \\
\hline First 8 months of data & $2.21 \pm 0.04_{\text {stat }} \pm 0.06_{\text {syst }}$ & $6.3 \pm 1.1_{\text {stat }} \pm 0.4_{\text {syst }}$ & $0.82 \pm 0.03_{\text {stat }} \pm 0.07_{\text {syst }}$ \\
\hline 30 months of data & $2.07 \pm 0.02_{\text {stat }} \pm 0.09_{\text {syst }}$ & $3.9 \pm 0.2_{\text {stat }} \pm 0.7_{\text {syst }}$ & $0.95 \pm 0.01_{\text {stat }} \pm 0.07_{\text {syst }}$ \\
\hline Data before 2009 March & $2.08 \pm 0.04_{\text {stat }} \pm 0.09_{\text {syst }}$ & $4.0 \pm 0.6_{\text {stat }} \pm 0.7_{\text {syst }}$ & $0.75 \pm 0.03_{\text {stat }} \pm 0.07_{\text {syst }}$ \\
\hline Data after 2009 March & $2.07 \pm 0.02_{\text {stat }} \pm 0.09_{\text {syst }}$ & $3.9 \pm 0.3_{\text {stat }} \pm 0.7_{\text {syst }}$ & $1.00 \pm 0.01_{\text {stat }} \pm 0.07_{\text {syst }}$ \\
\hline Inferior conjunction (geometrically) & $2.14 \pm 0.02_{\text {stat }} \pm 0.09_{\text {syst }}$ & $4.0 \pm 0.4_{\text {stat }} \pm 0.7_{\text {syst }}$ & $1.07 \pm 0.02_{\text {stat }} \pm 0.07_{\text {syst }}$ \\
\hline Superior conjunction (geometrically) & $2.02 \pm 0.03_{\text {stat }} \pm 0.09_{\text {syst }}$ & $3.9 \pm 0.3_{\text {stat }} \pm 0.7_{\text {syst }}$ & $0.85 \pm 0.02_{\text {stat }} \pm 0.07_{\text {syst }}$ \\
\hline Inferior conjunction (angle cut) & $2.17 \pm 0.03_{\text {stat }} \pm 0.09_{\text {syst }}$ & $4.1 \pm 0.5_{\text {stat }} \pm 0.7_{\text {syst }}$ & $1.11 \pm 0.03_{\text {stat }} \pm 0.07_{\text {syst }}$ \\
\hline Superior conjunction (angle cut) & $2.15 \pm 0.03_{\text {stat }} \pm 0.09_{\text {syst }}$ & $5.0 \pm 0.7_{\text {stat }} \pm 0.7_{\text {syst }}$ & $0.91 \pm 0.02_{\text {stat }} \pm 0.07_{\text {syst }}$ \\
\hline Periastron & $2.14 \pm 0.02_{\text {stat }} \pm 0.09_{\text {syst }}$ & $4.1 \pm 0.4_{\text {stat }} \pm 0.7_{\text {syst }}$ & $1.01 \pm 0.02_{\text {stat }} \pm 0.07_{\text {syst }}$ \\
\hline Apastron & $2.01 \pm 0.03_{\text {stat }} \pm 0.09_{\text {syst }}$ & $3.7 \pm 0.3_{\text {stat }} \pm 0.7_{\text {syst }}$ & $0.90 \pm 0.02_{\text {stat }} \pm 0.07_{\text {syst }}$ \\
\hline
\end{tabular}

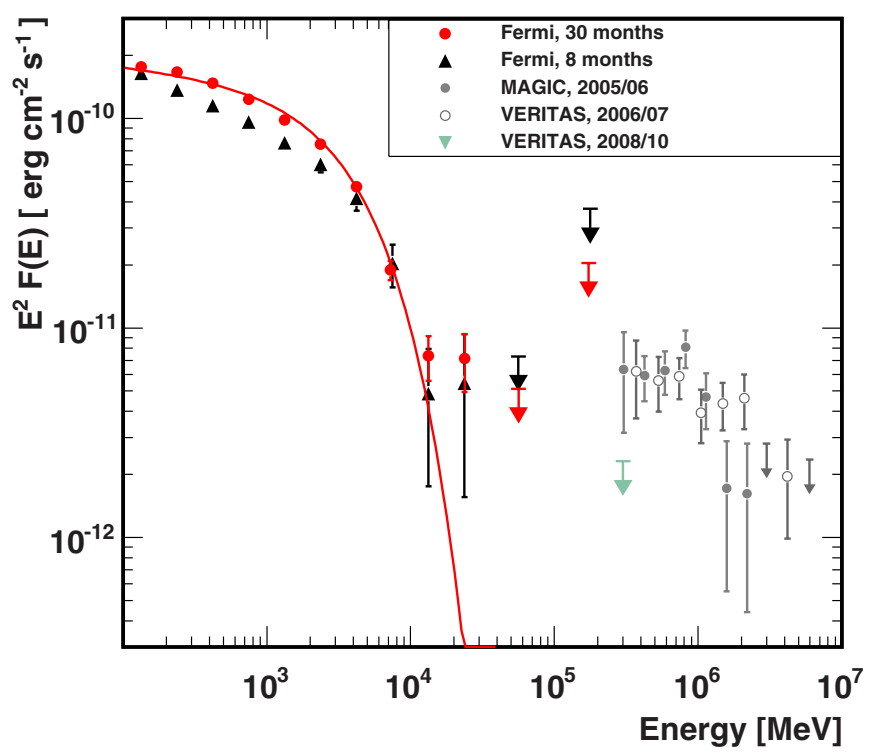

Figure 4. Overall 30 month LS I $+61^{\circ} 303$ spectrum (red) in comparison with the earlier published one (black) over 8 months is shown. TeV data points taken by MAGIC and VERITAS are shown in gray. They are not simultaneously taken with the $\mathrm{GeV}$ data, whereas the data for the VERITAS upper limit (in green) are.

(A color version of this figure is available in the online journal.)

and corresponding best fit using the updated data set described in Section 2 are shown in Figure 4, together with previously derived results from the LAT and TeV observations. Two sets of $\mathrm{TeV}$ data are plotted: we show the non-simultaneous data points obtained by the Cherenkov telescope experiments MAGIC and VERITAS. (These data correspond to phases around 0.6-0.7 and represent several orbits observed in the period 2006-2008, before Fermi was launched.) Additionally, we show the latest measurements performed by VERITAS, which established a 99\% CL upper limit. ${ }^{14}$ The new VERITAS upper limit spans several orbits during which, simultaneously with our LAT data, no detection was achieved. The LAT data along the whole orbit are still best described by a power law with an exponential cutoff. The $T S$ value for a source emitting $\gamma$-rays at the position of $\mathrm{LS} \mathrm{I}+61^{\circ} 303$ with an SED described by a power law with an exponential cutoff is highly significant. The relative $T S$ value comparing a fit with a power law and a fit with a power law plus an exponential cutoff clearly favors the latter, at the $\sim 20 \sigma$ level. The photon index found is $\Gamma=2.07 \pm 0.02_{\text {stat }} \pm 0.09_{\text {syst }}$; the flux above $100 \mathrm{MeV}$ is $\left(0.95 \pm 0.01_{\text {stat }} \pm 0.07_{\text {syst }}\right) \times 10^{-6}$ photons $\mathrm{cm}^{-2} \mathrm{~s}^{-1}$, and the cutoff energy is $3.9 \pm 0.2_{\text {stat }} \pm 0.7_{\text {syst }} \mathrm{GeV}$. Results for the obtained $T S$ values for each fit to different data sets are listed in Table 1 and all fit parameters obtained for the exponentially cutoff power-law models are listed in Table 2.

Figure 4 shows that the data point at $30 \mathrm{GeV}$ deviates from the model by more than $3 \sigma$ (power law with cutoff, red line). Although in our representation it is only one point, it is in itself significant, with a $T S$ value of 67 corresponding to $\sim 8 \sigma$. Therefore, and similar to the case of LS 5039, with the caveat of having only one point determined in the SED beyond the results of the fitted spectral model, we investigate the possible presence of a second component at HEs. As in the case for LS 5039, we use the likelihood ratio test to compare two models: model $\mathrm{A}$ is a power law with a cutoff and model $\mathrm{B}$ is a power law with a cutoff plus an additional power law. According to this test, the probability of incorrectly rejecting model A is $5.7 \times 10^{-15}(7.8 \sigma)$. The $T S$ value for this extra

\footnotetext{
14 We derive this differential upper limit by using the VERITAS-reported integral flux upper limit for phases 0.6-0.7 (Acciari et al. 2011) assuming a differential spectral slope of 2.6 .
} 


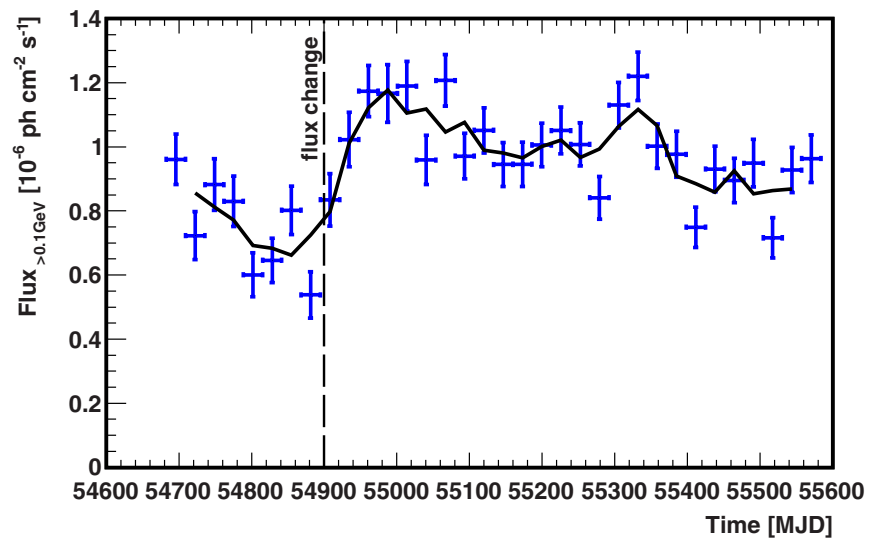

Figure 5. Light curve of LS I $+61^{\circ} 303$. Each point represents one orbit of 26.496 days, whereas the black solid line represents the three-bin-smoothed light curve. The black dashed line marks the moment of the flux change in 2009 March.

(A color version of this figure is available in the online journal.)

power-law component as a whole is 172 , larger than in the case of LS 5039, and its parameters are $\Gamma=2.5 \pm 0.3_{\text {stat }}$ and $F_{>10 \mathrm{GeV}}=\left(3.5 \pm 0.6_{\text {stat }}\right) \times 10^{-10} \mathrm{~cm}^{-2} \mathrm{~s}^{-1}$. The addition of the HE component affects the parameters of the cutoff power law. The best-fit parameters for the latter, when including the former, are $\Gamma=2.00 \pm 0.03_{\text {stat }}$ and $E_{\text {cutoff }}=2.7 \pm 0.3_{\text {stat }} \mathrm{GeV}$ with a flux of $F_{>100 \mathrm{MeV}}=\left(0.88 \pm 0.08_{\text {stat }}\right) \times 10^{-6} \mathrm{~cm}^{-2} \mathrm{~s}^{-1}$. Compared with the cutoff energy of $3.9 \pm 0.2 \mathrm{GeV}$ we obtain after fitting only a power law with an exponential cutoff to the data, the cutoff energy decreases when the additional HE power-law component is introduced.

An alternative model to accommodate the deviating $\mathrm{HE}$ point in the spectrum is a fit with a broken power law. This is further discussed in Section 4.4.

\subsection{Light Curve}

In Figure 5, the light curve for LS I +61 303 over 30 months is shown using orbital time bins. The black dashed line represents the point in time when a flux change occurred for LS I +61 303; this occurred in 2009 March. In Figure 6, we present the power spectrum of LS I $+61^{\circ} 303$ derived from the total weighted photon light curve. The power spectrum clearly detects the orbital flux modulation with a period of $26.71 \pm$ 0.05 days. This is consistent with the known orbital period of LS I $+61^{\circ} 303$ (Gregory 2002). The light curve clearly shows long-term variability. We searched for changes in the orbital modulation of $\mathrm{LS} \mathrm{I}+61^{\circ} 303$ by dividing the aperture photometry light curves into six month segments and calculating the power spectrum of each segment, as shown in the left panel of Figure 7. The amplitude of the orbital modulation is estimated by fitting a sine wave fixed to the orbital period to each of the light curve segments; the results, as seen in the right panel of Figure 7 , clearly show a decreasing trend in the orbital modulation with time.

LS I $+61^{\circ} 303$ is one of the brightest sources in the $\gamma$-ray sky and towers above all other emitters in its neighborhood. This allows us to compute a light curve with an orbital binning (26.496 days per bin) which is shown in Figure 5. Even by eye, it is clear that the source is highly variable on orbital timescales and longer. The longer term trends are evident by looking at a plot of the three-orbit rolling average (black line in Figure 5). During the first eight orbits, the flux decreases by a factor of 2. Then, in 2009 March, the flux appears to increase over

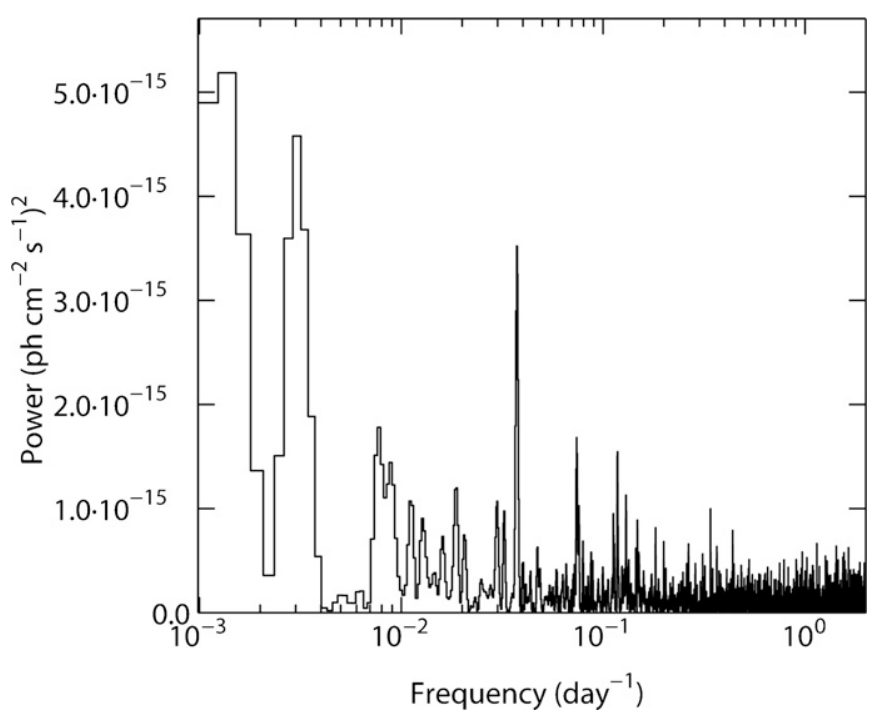

Figure 6. Lomb-Scargle periodogram of the whole LS I +61 $303 \mathrm{GeV}$ data set. The orbital period is clearly visible.

the course of several orbits; we take the transition point of this increase to be MJD 54,900.

The flux increases significantly by $33 \% \pm 4 \%$, rising from a baseline of $\left(0.75 \pm 0.03_{\text {stat }} \pm 0.07_{\text {syst }}\right) \times 10^{-6}$ photons cm $\mathrm{cm}^{-2} \mathrm{~s}^{-1}$ obtained from the first eight months of data to $\left(1.00 \pm 0.01_{\text {stat }} \pm\right.$ $\left.0.07_{\text {syst }}\right) \times 10^{-6}$ photons $\mathrm{cm}^{-2} \mathrm{~s}^{-1}$ which is the average flux of the remaining 1.7 years of the data. Comparing the flux levels averaged over the same time span, eight months before and eight months after the flux change, we obtain a 40\% increase. After this flux change, the flux decreases again slowly over the remaining 1.7 years. The complexities of the short timescale, orbit-to-orbit variability make it impossible to characterize the exact properties of the transition from the "lower" to "higher" flux states. The transition likely took place over several orbits, however, for simplicity throughout the remainder of this analysis we use a transition time of MJD 54,900.

We graphically show the flux change in Figure 8, by plotting the folded light curves before and after the transition in 2009 March. The data points are folded on the Gregory (2002) period, with zero phase at MJD 43,366.775. Before the transition, the modulation was clearly seen and is compatible with the already published phasogram, whereas afterward, the amplitude of the modulation diminishes. We quantify this behavior by measuring the flux fraction below. Note that the data sets corresponding to the reported results (Abdo et al. 2009a) and what we here referred to as before the flux change span almost exactly the same time range, with the consequence of our current analysis essentially reproducing that previously published. The time span covered by our earlier publication coincidentally finished just prior to the onset of the flux change. The spectra derived before and after this flux change are shown in Figure 8, where the increase in flux is also obviously visible.

\subsection{Phase-resolved Spectral Analysis}

The statistics of the current data set allow us to divide the orbit into different phase ranges and to compute the corresponding spectra for different phase bins. We have divided the orbit into INFC and SUPC phase ranges in two different ways. First, we have split the LS I $+61^{\circ} 303$ orbit in two halves based on its geometry, as visualized, for instance, in Aragona et al. (2009). The SUPC phase range is defined as from phase 0.63 to phase 

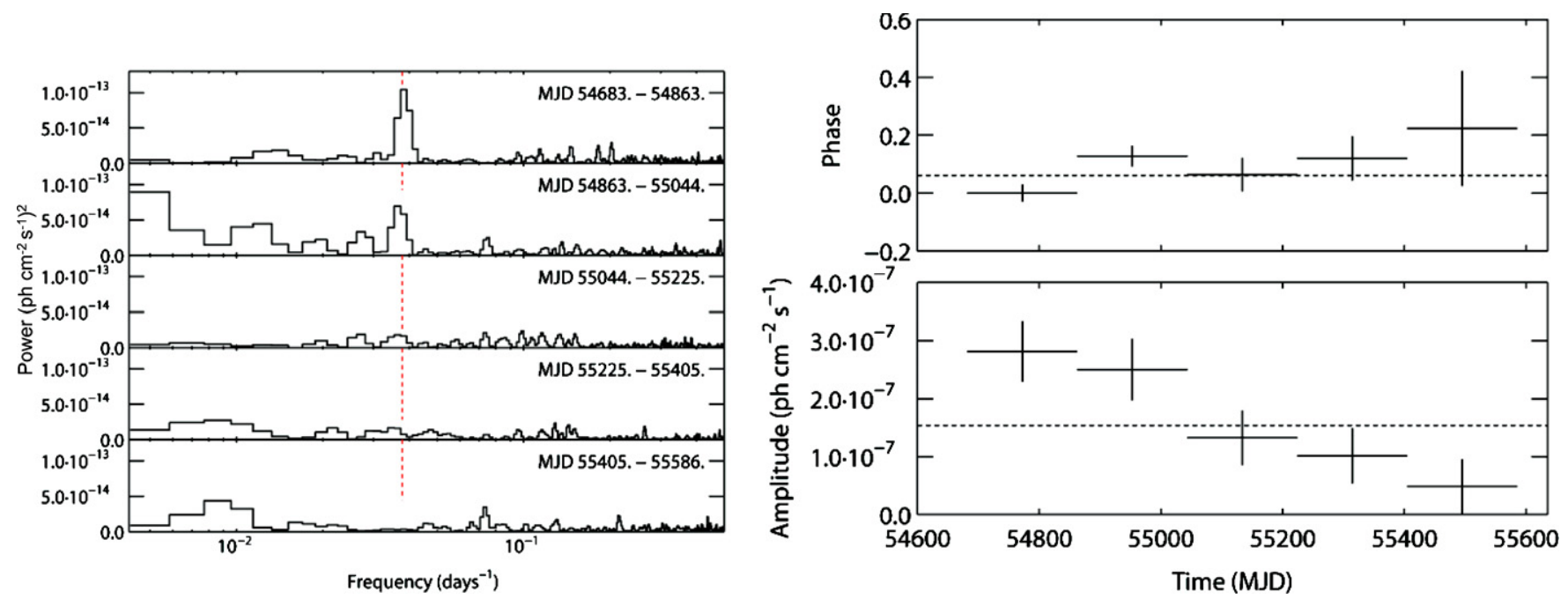

Figure 7. Left: the Lomb-Scargle periodograms of the LS I $+61^{\circ} 30330$ month light curve split into five consecutive segments; the earliest is at the top. The red dashed line indicates the known orbital period. Right: the change in the amplitude and phase of the orbital flux modulation calculated by fitting a sine wave to each of the light curve segments.

(A color version of this figure is available in the online journal.)
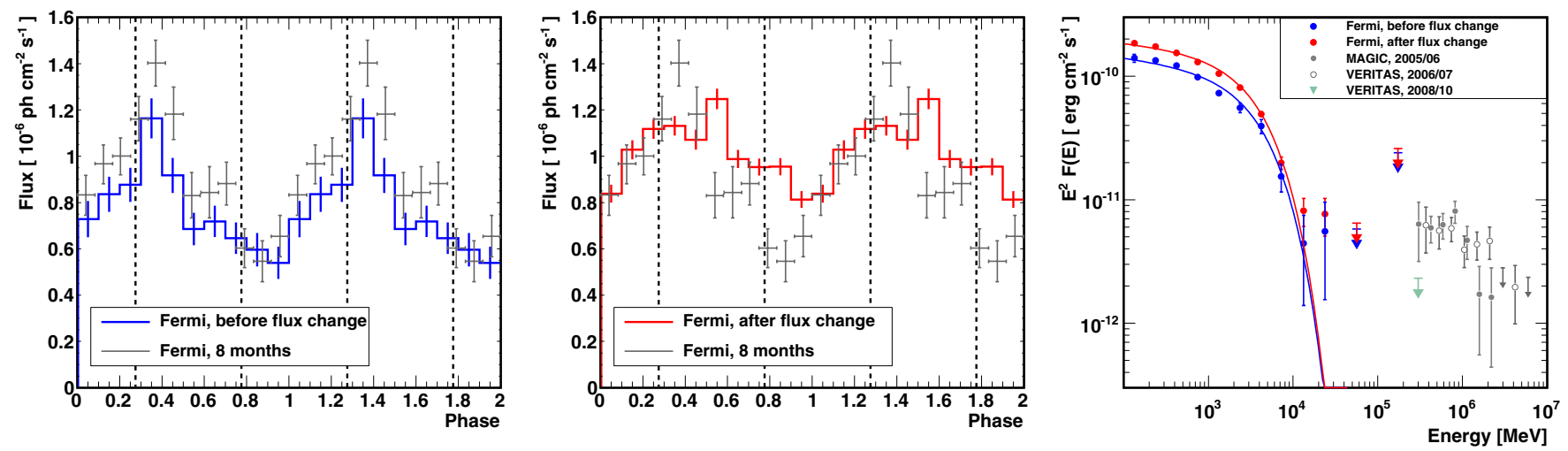

Figure 8. Left: folded light curve ( $100 \mathrm{MeV}-300 \mathrm{GeV})$ of LS I $+61^{\circ} 303$ before the flux change (blue), when the modulation is still clearly visible, compared with the earlier published eight month data set shown in gray. Middle: folded light curve (100 MeV-300 GeV) after the flux change, in 2009 March (red). The modulation gets fainter. For comparison, the previous published light curve is also plotted in gray. Right: comparison of the spectra derived before (blue) and after (red) the flux change in 2009 March.

(A color version of this figure is available in the online journal.)
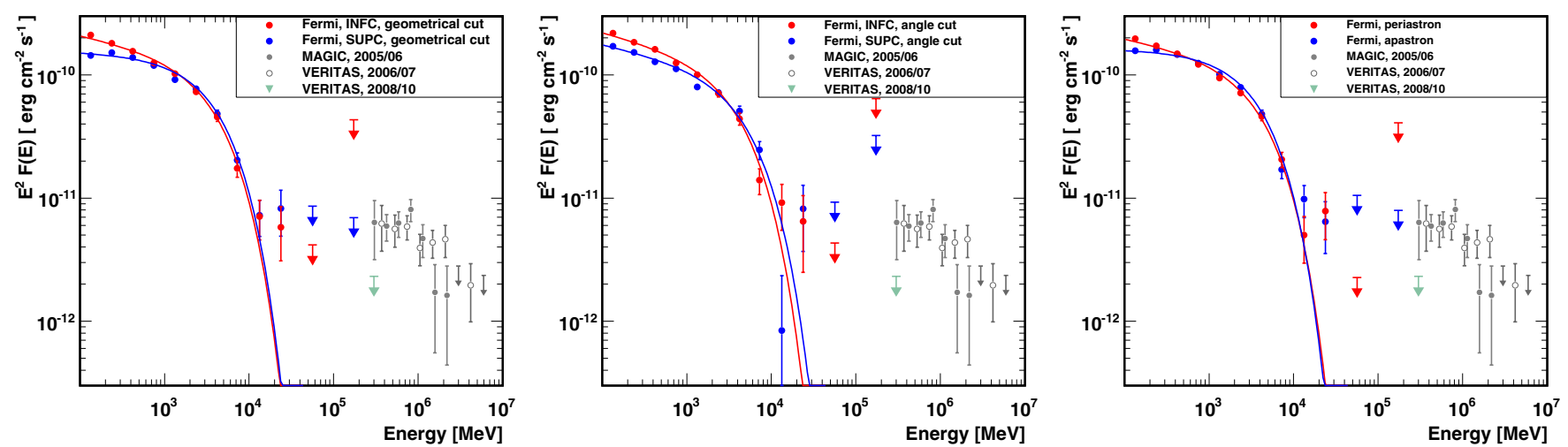

Figure 9. Spectra of LS I $+61^{\circ} 303$ for different phase bins during the orbit are shown. Left: spectra for the geometrical cut of the orbit in superior (blue) and inferior (red) conjunction. Middle: spectra for the angle-based cut in superior and inferior conjunction. Right: spectra derived for periastron (red) and apastron (blue).

(A color version of this figure is available in the online journal.)

0.13 ; INFC is defined correspondingly, as the remaining half. We also adopted another way to separate between INFC and SUPC based on the angle between the compact object, the star, and the observer. Therefore, the orbit is not divided in two halves in this way, but in one piece when the compact object is in front of the star (corresponding to INFC): $0.244-0.507$; and in another phase range with the same duration, centering at the exact SUPC phase: 0.981-0.244. As can be seen in Figure 9, the spectra obtained with the different cuts do not differ significantly and all spectral parameters are compatible within their corresponding 


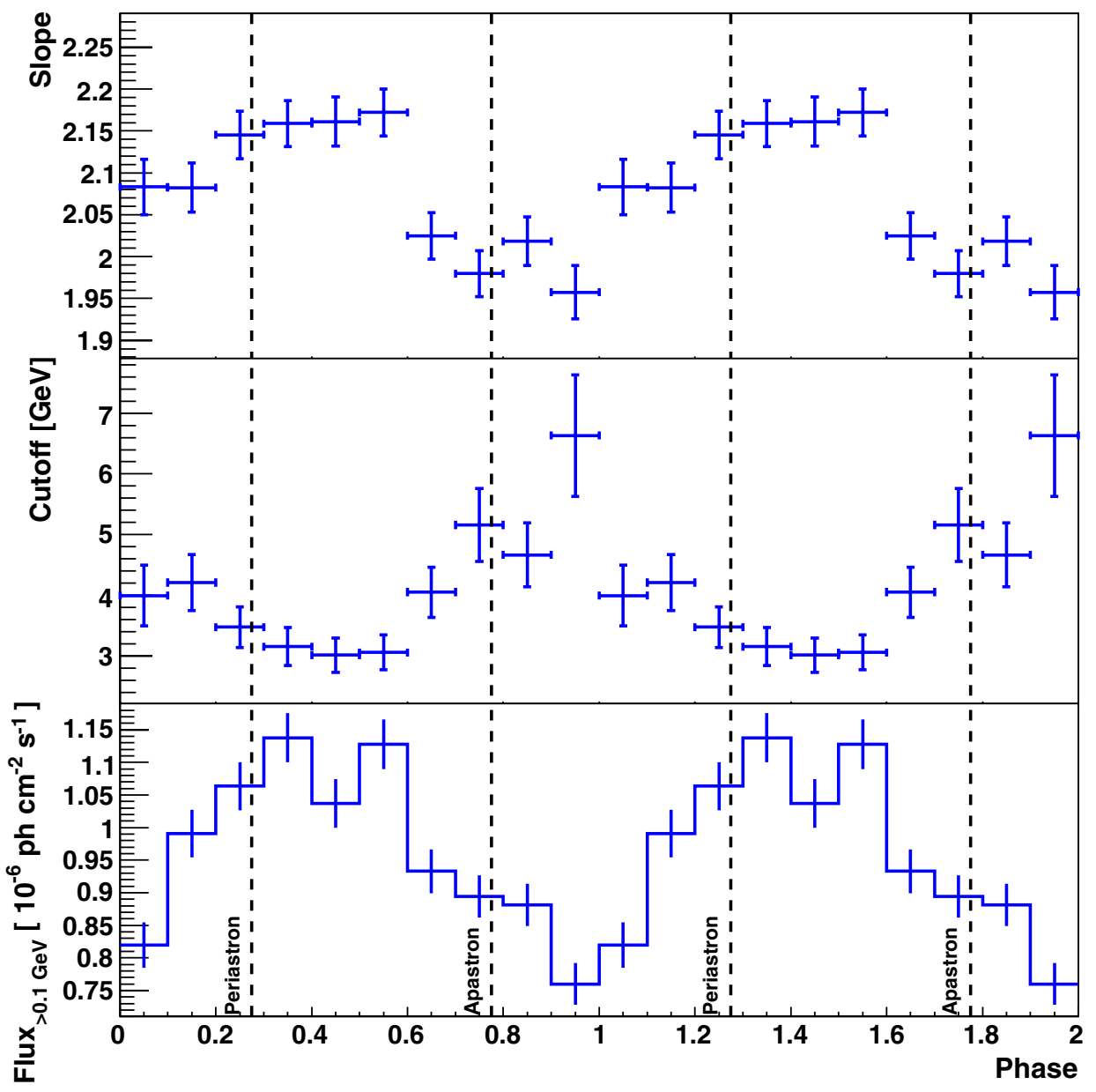

Figure 10. Modulation of the spectral parameters of LS I $+61^{\circ} 303$ on the total 30 month light curve. For details see Section 4.3.

(A color version of this figure is available in the online journal.)

errors (see Table 2). We find that the flux difference between INFC and SUPC is of the order of $20 \%$. For these different data sets, representing only portions of the orbit, we modeled the source with a pure power law and with a power law with an exponential cutoff. The $\Delta T S$ value comparing both fits for the angular based cut is 108 (INFC), which means that the probability of incorrectly rejecting a power law with respect to an exponential cutoff is $2.8 \times 10^{-25}(10 \sigma)$. For SUPC the $\Delta T S$ value is 97 , which leads to a probability of $6.9 \times 10^{-12}(6.8 \sigma)$ to wrongly reject the cutoff power law. Hence, the exponential cutoff is preferred over the pure power law also for two parts of the orbit, namely, INFC or SUPC.

We have also divided the orbit into phase ranges corresponding to periastron (half of the orbit around phase 0.275) and apastron (the other half, around phase 0.725 ), based just on the distance between the compact object and the star. In this case, neither a significant difference between the flux values for the two phase bins nor a difference in the spectral shape is visible. These results can also be seen in Figure 9. This is probably the result of dividing the orbit into phase ranges which contain both bin phases corresponding to INFC and SUPC.

We also studied the spectral behavior of the source in phase bins of 0.1 . For this study, we modeled LS I $+61^{\circ} 303$ with a power law with a cutoff for each phase bin individually. The spectral parameters obtained are shown in Figure 10. Note that we fixed in the model the index at 2.07 to study the orbital behavior of the cutoff energy and we fixed the cutoff energy at
3.9 $\mathrm{GeV}$ to study the behavior of the index, since both parameters are correlated. These values are, respectively, the results arising from the fit over the whole data set. A clear orbital modulation of the flux and spectral shape is seen. Through the periastron passage the spectrum gets softer and the flux is maximum, whereas around apastron the spectrum becomes harder and the flux reaches its minimum.

\subsection{Spectral Fitting}

Both the orbitally averaged spectrum and the spectra of the several data sets mentioned were also fitted with a broken power law, in addition to the pure and exponentially cutoff power law. All the $T S$ values for the different fits are listed in Table 1. It is evident that the power law with an exponential cutoff or the broken power law always describe the spectral shape better than a pure power law does. To be precise, when comparing the exponentially cutoff power law with the pure power law, the $\Delta T S$ values span the range from 90 to 520 ; with the former always being statistically preferred. Fitting a broken power law gives almost the same results. The $\Delta T S$ values span the range from 77 to 494 and we find break energies in the range of $0.4-1.7 \mathrm{GeV}$. All of the fits to the various data sets with an exponentially cutoff power law have slightly better $T S$ values with fewer degrees of freedom than a broken power law, suggesting that the former model is a better description of the data than the latter one. The exponentially cutoff power 

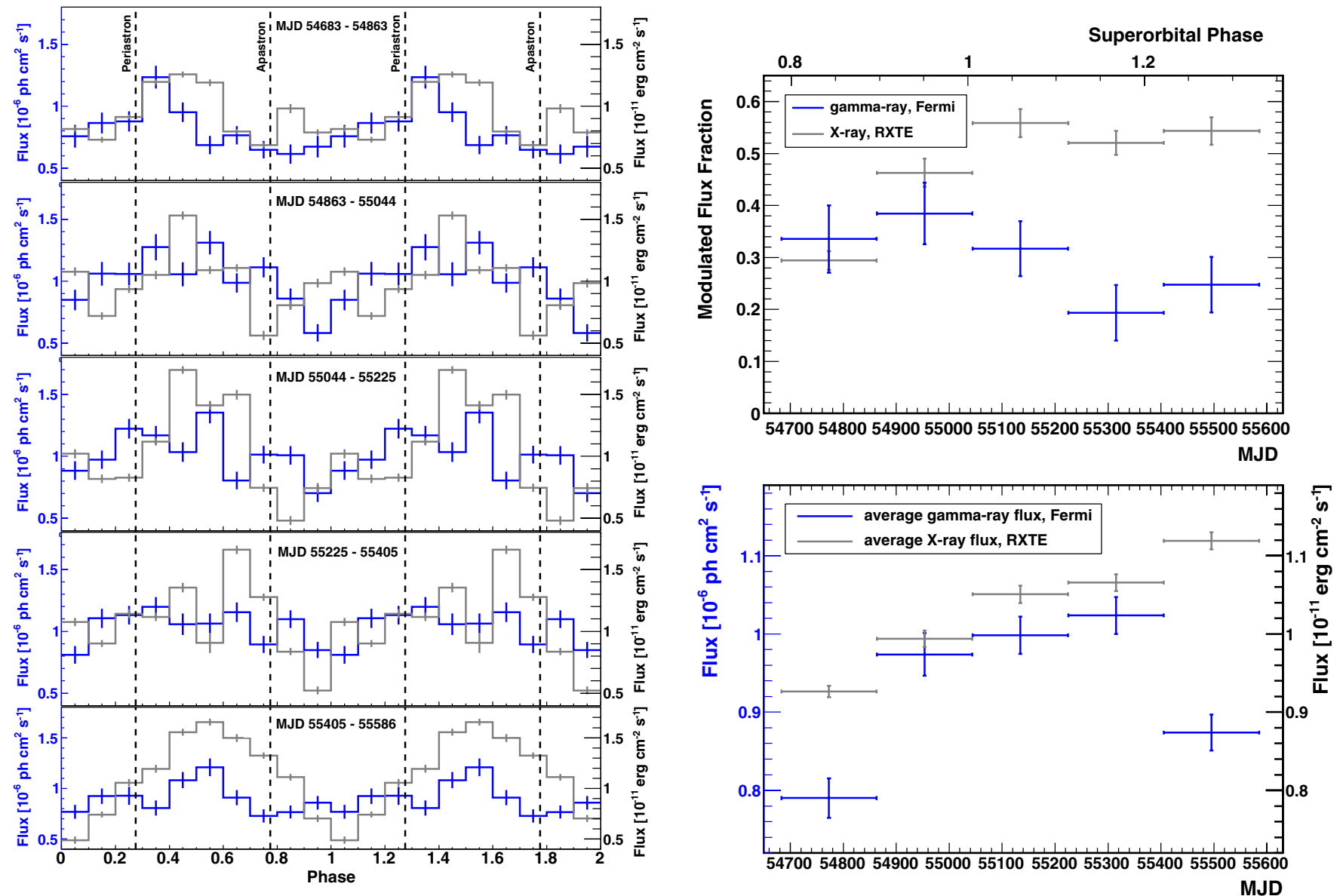

Figure 11. Comparison between the $\gamma$-ray (blue) and the X-ray (gray) data of LS I $+61^{\circ} 303$. Left: for each of the five separate six month periods, the $3-10 \mathrm{keV}$ (gray) and the $100 \mathrm{MeV}-300 \mathrm{GeV}$ (blue) folded light curves are shown. Right: six month modulated flux fraction data in both energy bands, with equal color coding.

(A color version of this figure is available in the online journal.)

law nicely describes the curvature of the spectrum, especially at low energies. At HEs, above the spectral break, the broken power-law fits all the spectral points, even the highest one which might possibly be considered part of a second component. Statistically, we cannot distinguish which of these two fitting models describes best the data as a whole, but only that both of them are preferred over a pure power law.

\subsection{The Multi-wavelength Context}

$$
\text { 4.5.1. X-Rays }
$$

LS I $+61^{\circ} 303$ has also been monitored with the $R X T E$-Proportional Counter Array (PCA) and folded light curves were produced using the same ephemeris as described in Section 4.2. In the left panel of Figure 11, we show a direct comparison between the phasograms in X-ray and in $\gamma$-rays, with simultaneously taken data. We divide the whole LAT data set into five periods of six months each and compare them with correspondingly obtained PCA X-ray data. The division into periods of six months is justified in order to have enough statistics in $\gamma$-rays for each individual time bin, such that orbit-to-orbit $\mathrm{X}$-ray variability does not dominate the flux fraction changes.

For RXTE-PCA, we have used the "Standard 2" mode for spectral analysis. Data reduction was performed using HEASoFT 6.9. We select time intervals where the source elevation above Earth limb is $>10^{\circ}$ and the pointing offset is $<0.02$. PCA background light curves and spectra were generated using the FTOOLS task pcabackest. pcarsp was used to generate PCA response matrices for spectra. The background file used in the analysis of PCA data is the most recent available from the HEASARC Web site for faint sources, and detector breakdown events have been removed. ${ }^{15}$ A powerlaw shape, with absorbing hydrogen column density fixed at $0.75 \times 10^{22} \mathrm{~cm}^{-2}$ (Kalberla et al. 2005; Smith et al. 2009), was used to fit the Standard 2 data, with the following function $N(E)=K e^{-N_{H} \sigma(E)}(E / \mathrm{keV})^{-\alpha}$, where $K$ is a normalization at $1 \mathrm{keV}, \sigma$ is the photoelectric cross-section, and $\alpha$ is the photon index.

It is apparent that the X-ray modulation is always visible in each of these five panels, albeit with variable amplitude of flux modulation. We define the flux fraction as $\left(c_{\max }-c_{\min }\right) /\left(c_{\max }+\right.$ $\left.c_{\min }\right)$, where $c_{\max }$ and $c_{\min }$ are the maximum and minimum flux in the $3-10 \mathrm{keV}$ found in the orbital profile analyzed (after background subtraction). The modulation becomes even stronger over time and stays stable over the last three half-year bins. Instead, at $\mathrm{GeV}$ energies, the LAT data indicate that the modulated fraction fades away until the variability along the orbit is barely visible in the last six months of our data, which is consistent with Figure 7. The flux fraction is plotted in the right

\footnotetext{
15 The background file is pca_bkgd_cmfaint17_eMv20051128.mdl and see the Web site: http://heasarc.gsfc.nasa.gov/docs/xte/recipes/pca_breakdown.html for more information on the breakdowns. The data have been barycentered using the FTOOLS routine faxbary using the JPL DE405 solar system ephemeris.
} 

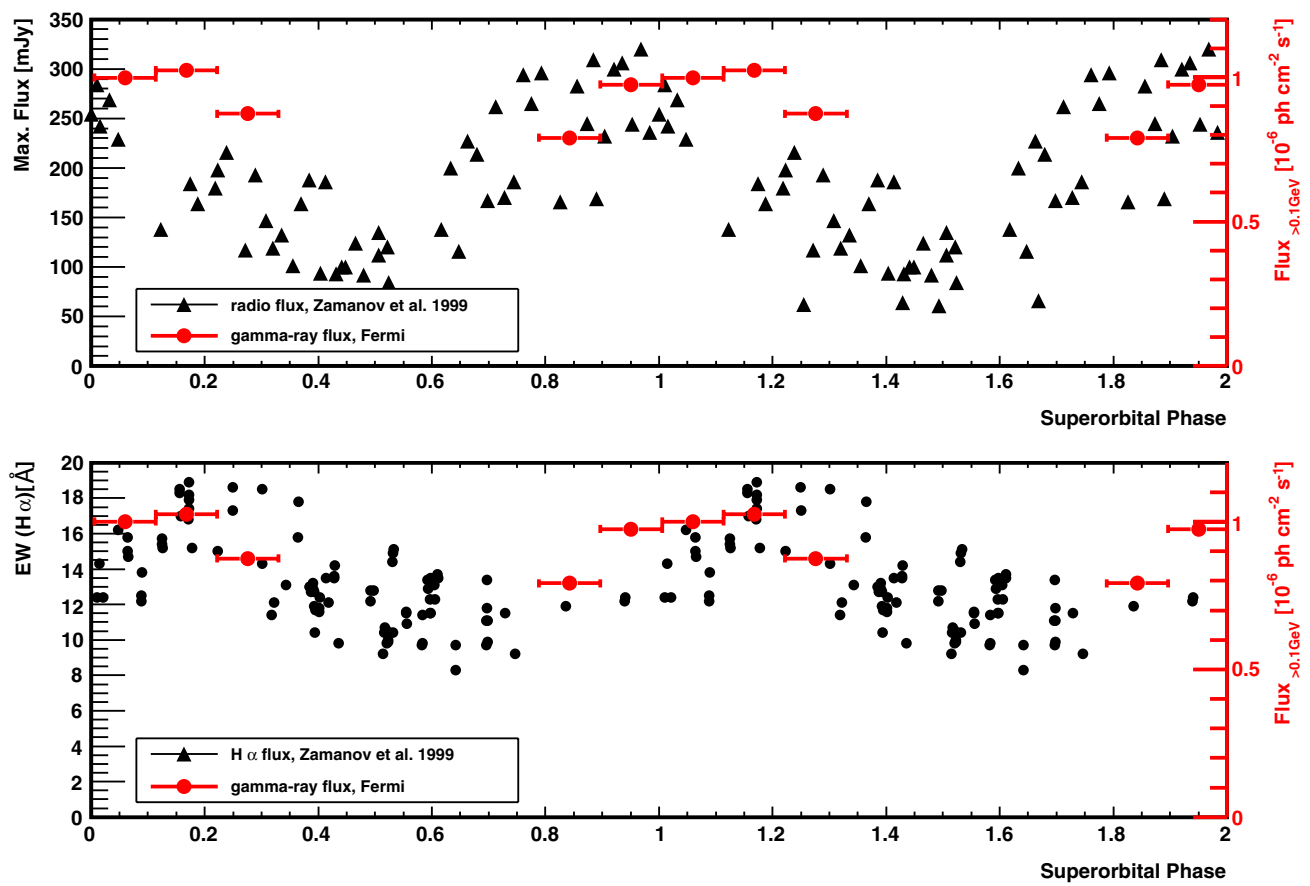

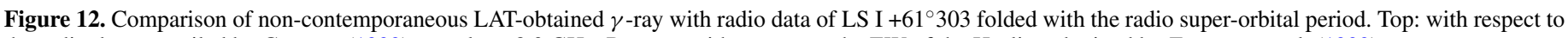
the radio data compiled by Gregory (1999), mostly at $8.3 \mathrm{GHz}$. Bottom: with respect to the EW of the H $\alpha$ line obtained by Zamanov et al. (1999).

(A color version of this figure is available in the online journal.)

top panel of Figure 11. The difference between the behavior of the X-ray and the $\gamma$-ray emission is clearly visible. In the right bottom panel of Figure 11, we show the average $\gamma$ - and X-ray fluxes fitted in each of the periods considered. We checked for a possible appearance of the super-orbital periodicity $1667 \pm$ 8 days, taking zero phase at MJD 43,366.275 and the shape of the outburst peak flux modulation estimated by Gregory (2002) in radio by considering the flux evolution and the direct count rate, using the full data set of the PCA observations. We find no evidence of the super-orbital period in X-ray or $\gamma$-rays, which is not surprising given the short integration time in comparison with the super-orbital period duration.

\subsubsection{Radio and Optical}

We have also compared the LAT-obtained data with radio and $\mathrm{H} \alpha$ observations. For the latter, we first take into account the results contained in the long-term coverage presented by Zamanov et al. (1999). These authors have already shown that the equivalent width (EW) and the peak separation of the $\mathrm{H} \alpha$ emission line appear to vary with the super-orbital radio period of $\sim 1600$ days, this likely being the result of cyclical variations in the mass-loss rate of the Be companion and/or of density variability in the circumstellar disk. Zamanov et al. (1999) proposed that the variability in the $\mathrm{EW}(\mathrm{H} \alpha)$ can be explained by a cyclical change in the mass-loss rate of about $25 \%$ over its average value. This mechanism would imply changes in the density of the circumstellar disk in the same range. The fact that the $\gamma$-ray light curve would have a similar behavior to that found for the $\mathrm{EW}(\mathrm{H} \alpha)$ would imply that the former is related to the circumstellar disk surrounding the Be star. This would naturally be the case if the $\gamma$-rays are produced in an inter-wind shock formed by the collision of outflows from the compact object and the star itself. Dubus (2006) and Sierpowska-Bartosik \& Torres (2009) discussed how the many uncertainties present in our knowledge of the $\mathrm{LS} \mathrm{I}+61^{\circ} 303$ system influence the models for producing $\gamma$-ray emission. One of the important parameters is the density and size (and the possible truncation) of the circumstellar disk. The latter influence the position of the inter-wind shock, and the time intervals along the orbit in which the compact object outflow may be balanced by the equatorial wind feeding it, and/or when the compact object is directly within the circumstellar disk itself. For recent measurements on very long term optical variability of Be high-mass X-ray binaries in the Small Magellanic Cloud, see Rajoelimanana et al. (2011).

We plot in Figure 12 a comparison of the LAT data, folded on the super-orbital radio period, with the radio data compiled by Gregory (1999)—most of it obtained at $8.3 \mathrm{GHz}$, using the Green Bank Interferometer-and the EW of the $\mathrm{H} \alpha$ line obtained by Zamanov et al. (1999). Care should be exercised when comparing our LAT data with the radio data in Zamanov et al. (1999), since the latter authors used the ephemeris given by Gregory (1999), for which the combination of the phase and peak flux density yielded a best-modulation period of 1584 days. This super-orbital period was later revised by Gregory (2002) to the current value of 1667 days. This latter value is the superorbital period that we have used to fold the X-ray and LAT data, and we also use it here for the radio and $\mathrm{H} \alpha$ emission. The comparison is clearly non-contemporaneous, and this may induce problems in the interpretation of a source like LS I $+61^{\circ} 303$, presenting different variability phenomenology. We do not see any clear correlation or anti-correlation with the radio and $\mathrm{H} \alpha$ fluxes which may be hidden by the scarcity of $\mathrm{GeV}$ data.

We have also compared the Fermi data with simultaneous radio data from the Owens Valley Radio Observatory (OVRO) and the Arcminute Microkelvin Imager (AMI) array (Cambridge, UK). Since the launch of Fermi in 2008 June, the OVRO $40 \mathrm{~m}$ single-dish telescope, which is located in California (USA), has conducted a regular monitoring program of Galactic binaries (e.g., Cyg X-3; Abdo et al. 2009c). The OVRO flux densi- 

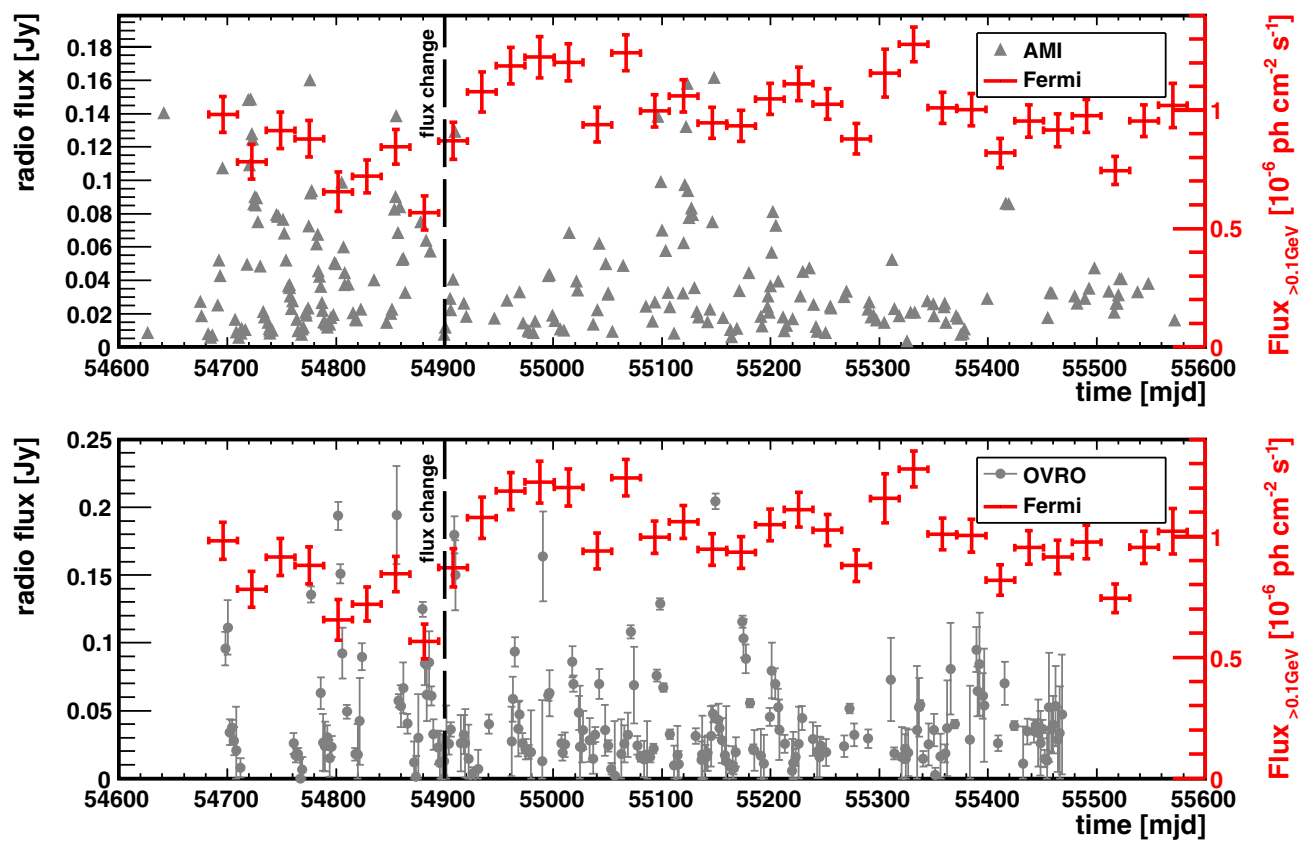

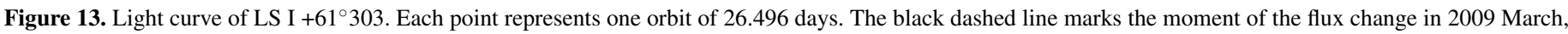
together with the simultaneously taken radio data by the AMI and OVRO instruments (both operating at $15 \mathrm{GHz}$ ) are shown in gray.

(A color version of this figure is available in the online journal.)

ties are measured in a single $3 \mathrm{GHz}$ wide band centered on $15 \mathrm{GHz}$. A complete description of the OVRO $40 \mathrm{~m}$ telescope and calibration strategy can be found in Richards et al. (2011). Furthermore, we also use complementary observations provided by AMI, consisting of a set of eight $13 \mathrm{~m}$ antennas with a maximum baseline of $\sim 120 \mathrm{~m}$. AMI observations are conducted with a $6 \mathrm{GHz}$ bandwidth receivers also centered at $15 \mathrm{GHz}$. See Zwart et al. (2008) for more details on the AMI interferometer, which is mostly used for the study of the cosmic microwave background. By folding these radio data, no super-orbital modulation could be seen, which could be due to the poor coverage of a whole super-orbit. A direct comparison of the $\mathrm{GeV}$ and the radio data is shown in Figure 13 where the flux over time is plotted. No correlation of the data points can be seen either. In summary, we did not find any correlation between the $\mathrm{GeV}$ and the radio band, either in archived or in simultaneous data.

\section{CONCLUDING REMARKS}

After analyzing a data set comprising 2.5 years of Fermi-LAT observations of the two binaries LS 5039 and LS I +61 303 , we note several changes with respect to the initial reports of Abdo et al. (2009a, 2009b). These were produced either because the accumulation of a longer observation time allowed us to make distinctions that were earlier impossible (valid for both sources), or because the behavior of the source changed (valid for LS I $+61^{\circ} 303$ ). On one hand, the statistics are now sufficient to divide the data set of both sources in INFC and SUPC and to show that a power law with a cutoff describes the spectra obtained in both conjunctions better than a pure power law. The cutoff is similar to that found in the many other GeV pulsars discovered by Fermi-LAT. However, we have found that both LS 5039 and LS I +61 303 show an excess of the HE GeV emission beyond what is expected from an exponentially cutoff power law. While the HE data are significantly in excess of the exponentially cutoff power law, there are insufficient statistics at these HEs to model the excess with an additional spectral component.
The process(es) generating such a component in the cases of LS 5039 and LS I $+61^{\circ} 303$ is unclear and may even be different in the two sources. However, such a second component would present a possible connection between the $\mathrm{GeV}$ and $\mathrm{TeV}$ spectra in both sources. Collecting more data, and therefore more statistics, will allow us to prove or discard it in the future. The lack of data points at HEs also affects, particularly in the case of LS I $+61^{\circ} 303$, the distinction between an exponentially cut and a broken power law. Currently, both are certainly preferred over a pure power law, but differences in the significances provided between the former are minor.

We have noted that, whereas LS 5039 shows stable emission over time and also a stable orbital modulation, LS I +61 ${ }^{\circ} 303$ shows a change in flux in 2009 March. Afterward, the orbital modulation decreases (see the bottommost left panels of Figure 11) and the orbital period could not be detected in the $\mathrm{GeV}$ data. $\mathrm{LS} \mathrm{I}+61^{\circ} 303$ has also presented a complex, concurrent behavior at higher energies. At $\mathrm{TeV}$, for approximately the last two years, it seems to have been in a low state in comparison with the flux level that led to its discovery (Aleksić et al. 2011; Acciari et al. 2011). Additionally, it was detected once-after $4.2 \mathrm{hr}$ of observations - near the periastron, where the system was never seen at $\mathrm{TeV}$ energies before (Acciari et al. 2011). Both of these aspects of the LS I $+61^{\circ} 303$ phenomenology-as well as the phase location of the TeV maxima in general-would require modifications of simple inverse Compton models in the scenarios usually put forward for the source. The idea of a magnetar compact object in LS I $+61^{\circ} 303$ is suggested by Torres et al. (2012) to explain the changing TeV behavior of this object and could potentially describe the diminishing orbital modulation now observed at $\mathrm{GeV}$ fluxes. However, as explained by Torres et al. (2012), detailed numerical simulations are required to verify if this scenario can accurately reproduce the observed $\mathrm{GeV}$ and $\mathrm{TeV}$ emission.

The lower-energy, multi-wavelength picture of LS I $+61^{\circ} 303$ is still unclear, with the orbital modulation in $\mathrm{GeV}$ (X-ray) fading (increasing) with time. Because the $\mathrm{GeV}$ data do not cover 
a whole super-orbital period, a conclusion about the possible relationship of the $\mathrm{GeV}$ emission with the super-orbital behavior of LS I $+61^{\circ} 303$ cannot be drawn yet. Continued monitoring of the source with the Fermi-LAT will allow for the study of such long-term cycles, if any. Zamanov et al. (1999) concluded that if the $\sim 4$ year modulation in radio and $\mathrm{H} \alpha$ is due to changes in the circumstellar disk density, then it could be detected in X- or $\gamma$-rays as well, since the HE emission of a putative neutron star depends on the density of the surrounding matter with which it interacts. Indeed, this modulation was recently revealed in X-rays (Li et al. 2012).

The nature of these systems is thus still unclear and multiwavelength observations, including Fermi-LAT monitoring, as well as further theoretical studies are expected to bring new insights.

The Fermi LAT Collaboration acknowledges generous ongoing support from a number of agencies and institutes that have supported both the development and the operation of the LAT as well as scientific data analysis. These include the National Aeronautics and Space Administration and the Department of Energy in the United States, the Commissariat à l'Energie Atomique and the Centre National de la Recherche Scientifique/Institut National de Physique Nucléaire et de Physique des Particules in France, the Agenzia Spaziale Italiana and the Istituto Nazionale di Fisica Nucleare in Italy, the Ministry of Education, Culture, Sports, Science and Technology (MEXT), High Energy Accelerator Research Organization (KEK) and Japan Aerospace Exploration Agency (JAXA) in Japan, and the K. A. Wallenberg Foundation, the Swedish Research Council and the Swedish National Space Board in Sweden. Additional support for science analysis during the operations phase is gratefully acknowledged from the Istituto Nazionale di Astrofisica in Italy and the Centre National d'Études Spatiales in France. This work has been additionally supported by the Spanish CSIC and MICINN and the Generalitat de Catalunya, through grants AYA2009-07391 and SGR2009-811, as well as the Formosa Program TW2010005. S.Z. acknowledges supports from National Natural Science Foundation of China (via NSFC-10325313, 10521001, 10733010, 10821061, 11073021, and 11133002), and 973 program 2009CB824800. G.D. acknowledges support from the European Community via contract ERC-StG-200911. A.B.H. acknowledges funding via an EU Marie Curie International Outgoing Fellowship under contract no. 2010-275861. The AMI arrays are supported by STFC and the University of Cambridge.

\section{REFERENCES}

Abdo, A. A., Ackermann, M., Ajello, M., et al. 2009a, ApJ, 701, L123 Abdo, A. A., Ackermann, M., Ajello, M., et al. 2009b, ApJ, 706, L56 Abdo, A. A., Ackermann, M., Ajello, M., et al. 2009c, Science, 326, 1512 Abdo, A. A., Ackermann, M., Ajello, M., et al. 2010a, ApJS, 188, 405 Abdo, A. A., Ackermann, M., Ajello, M., et al. 2010b, ApJS, 187, 460
Abdo, A. A., Ackermann, M., Ajello, M., et al. 2011, ApJ, 736, L11 Acciari, V. A., Aliu, E., Arlen, T., et al. 2011, ApJ, 738, 3

Acciari, V. A., Beilicke, M., Blaylock, G., et al. 2008, ApJ, 679, 1427

Aharonian, F., Akhperjanian, A. G., Aye, K.-M., et al. 2005a, A\&A, 442, 1

Aharonian, F., Akhperjanian, A. G., Aye, K.-M., et al. 2005b, Science, 309, 746

Aharonian, F., Akhperjanian, A. G., Bazer-Bachi, A. R., et al. 2006, A\&A, 460, 743

Albert, J., Aliu, E., Anderhub, H., et al. 2006, Science, 312, 1771

Albert, J., Aliu, E., Anderhub, H., et al. 2007, ApJ, 665, L51

Albert, J., Aliu, E., Anderhub, H., et al. 2009, ApJ, 693, 303

Aleksić, J., Alvarez, E. A., Antonelli, L. A., et al. 2011, ApJ, 746, 80

Aleksić, J., Antonelli, L. A., Antoranz, P., et al. 2010, ApJ, 721, 843

Aragona, C., McSwaim, M. V., Grandstrom, E. D., et al. 2009, ApJ, 698, 514

Atwood, W. B., Abdo, A. A., Ackermann, M., et al. 2009, ApJ, 697, 1071

Barthelmy, S. D., Baumgartner, W., Cummings, J., et al. 2008, GCN Circ., 8215,1

Bednarek, W. 2007, A\&A, 464, 259

Bongiorno, S., Falcone, A., Stroh, M., et al. 2011, ApJ, 737, L11

Casares, J., Ribó, M., Ribas, I., et al. 2005, MNRAS, 364, 899

Corbet, R., Ackermann, M., Ajello, M., et al. 2012, Science, 335, 189

Corbet, R., \& Dubois, R. 2007, in AIP Conf. Proc. 921, The First GLAST Symposium, ed. S. Ritz, P. Michelson, \& C. A. Meegan (Melville, NY: AIP), 548

Dubus, G. 2006, A\&A, 456, 801

Dubus, G., Cerutti, B., \& Henri, G. 2008, A\&A, 477, 691

Eadie, W. T., Drijard, D., \& James, F. E. 1971, Statistical Methods in Experimental Physics (Amsterdam: North-Holland)

Falcone, A., Bongiorno, S., Stroh, M., \& Holder, J. 2011, ATel, 3152, 1

Gregory, P. C. 1999, ApJ, 520, 361

Gregory, P. C. 2002, ApJ, 575, 427

Hill, A. B., Dubois, R., Torres, D. F., et al. 2011, in Proceedings of the 1st Session of the Sant Cugat Forum of Astrophysics, High-Energy Emission from Pulsars and their Systems, ed. N. Rea \& D. F. Torres (Berlin: Springer), 498

Horne, J. H., \& Baliunas, S. L. 1986, ApJ, 302, 757

Kalberla, P. M. W., Burton, W. B., Hartmann, D., et al. 2005, A\&A, 440, 775 Kerr, M. 2011, ApJ, 732, 38

Khangulyan, D., Aharonian, F., \& Bosch-Ramon, V. 2008, MNRAS, 383, 467

Li, J., Torres, D. F., Zhang, S., et al. 2011, ApJ, 733, 89

Li, J., Torres, D. F., Zhang, S., et al. 2012, ApJ, 744, L13

Lomb, N. R. 1976, Ap\&SS, 39, 447

Mariotti, M. 2011, ATel, 3161, 1

Mattox, J. R., Bertsch, D. L., Chiang, J., et al. 1996, ApJ, 461, 396

Ong, R. A. 2010, ATel, 2948, 1

Ong, R. A. 2011, ATel, 3153, 1

Rajoelimanana, A. F., Charles, P. A., \& Udalski, A. 2011, MNRAS, 413, 1600

Ray, P. S., Kerr, M., Parent, D., et al. 2011, ApJS, 194, 17

Rea, N., Torres, D. F., Caliandro, G. A., et al. 2011, MNRAS, 416, 1514

Rea, N., Torres, D. F., van der Klis, M., et al. 2010, MNRAS, 405, 2206

Richards, J. L., Max-Moerbeck, W., Pavlidou, V., et al. 2011, ApJS, 194, 29

Sabatini, S., Tavani, M., Striani, E., et al. 2010, ApJ, 712, L10

Scargle, J. D. 1982, ApJ, 263, 835

Sierpowska-Bartosik, A., \& Torres, D. F. 2007, ApJ, 671, L145

Sierpowska-Bartosik, A., \& Torres, D. F. 2009, ApJ, 693, 1462

Smith, A., Kaaret, P., Holder, J., et al. 2009, ApJ, 693, 1621

Tam, P. H. T., Huang, R. H. H., Takata, J., et al. 2011, ApJ, 736, L10

Torres, D. F. 2011, in Proceedings of the 1st Session of the Sant Cugat Forum of Astrophysics, High-Energy Emission from Pulsars and their Systems, ed. N. Rea \& D. F. Torres (Berlin: Springer), 531

Torres, D. F., Rea, N., Esposito, P., et al. 2012, ApJ, 744, 106

Torres, D. F., Zhang, S., Li, J., et al. 2010, ApJ, 719, L104

Zamanov, R. K., Martí, J., Paredes, J. M., et al. 1999, A\&A, 351, 543

Zhang, S., Torres, D. F., Li, J., et al. 2010, MNRAS, 408, 642

Zwart, J. T. L., Barker, R. W., Biddulph, P., et al. 2008, MNRAS, 391, 1545 